\title{
The Italian autism network (ITAN): a resource for molecular genetics and biomarker investigations
}

Pierandrea Muglia ${ }^{*}$, Michele Filosi ${ }^{2}$, Lucio Da Ros ${ }^{3}$, Tony Kam-Thong ${ }^{4}$, Franco Nardocci ${ }^{5}$, Elisabetta Trabetti ${ }^{6}$, Emiliangelo Ratti ${ }^{7}$, Paolo Rizzini ${ }^{3}$, Alessandro Zuddas ${ }^{8}$, Bernardo Dalla Bernardina ${ }^{9}$, Enrico Domenici ${ }^{2,10^{*}}$ (D) and on behalf of the Italian Autism Network

\begin{abstract}
Background: A substantial genetic component accounts for Autism Spectrum Disorders (ASD) aetiology, with some rare and common genetic risk factors recently identified. Large collections of DNAs from thoroughly characterized ASD families are an essential step to confirm genetic risk factors, identify new variants and investigate genotype-phenotype correlations. The Italian Autism Network aimed at constituting a clinical database and a biorepository of samples derived from ASD subjects and first-degree relatives extensively and consistently characterized by child psychiatry centers in Italy.
\end{abstract}

Methods: The study was approved by the ethical committee of the University of Verona, the coordinating site, and by the local ethical committees of each recruiting site. Certified staff was specifically trained at each site for the overall study conduct, for clinical protocol administration and handling of biological material. A centralized database was developed to collect clinical assessment and medical records from each recruiting site. Children were eligible for recruitment based on the following inclusion criteria: age 4-18 years, at least one parent or legal guardian giving voluntary written consent, meeting DSM-IV criteria for Autistic Disorder or Asperger's Disorder or Pervasive Developmental Disorder NOS. Affected individuals were assessed by full psychiatric, neurological and physical examination, evaluation with ADI-R and ADOS scales, cognitive assessment with Wechsler Intelligence Scale for Children or Preschool and Primary, Leiter International Performance Scale or Griffiths Mental Developmental Scale. Additional evaluations included language assessment, the Krug Asperger's Disorder Index, and instrumental examination such as EEG and structural MRI. DNA, RNA and plasma were collected from eligible individuals and relatives. A central laboratory was established to host the biorepository, perform DNA and RNA extraction and lymphocytes immortalisation.

Discussion: The study has led to an extensive collection of biological samples associated with standardised clinical assessments from a network of expert clinicians and psychologists. Eighteen sites have received ADI/ADOS training, thirteen of which have been actively recruiting. The clinical database currently includes information on 812 individuals from 249 families, and the biorepository has samples for $98 \%$ of the subjects. This effort has generated a highly valuable resource for conducting clinical and genetic research of ASD, amenable to further expansion.

Keywords: Autism Spectrum disorders, Biomarkers, Biorepository, Genetics

\footnotetext{
*Correspondence: pierandrea.muglia@ucb.com; enrico.domenici@unitn.it

${ }^{1}$ UCB Pharma, Bruxelles, Belgium

${ }^{2}$ Centre for Integrative Biology, University of Trento, Trento, Italy

Full list of author information is available at the end of the article
}

(c) The Author(s). 2018 Open Access This article is distributed under the terms of the Creative Commons Attribution 4.0 International License (http://creativecommons.org/licenses/by/4.0/), which permits unrestricted use, distribution, and reproduction in any medium, provided you give appropriate credit to the original author(s) and the source, provide a link to the Creative Commons license, and indicate if changes were made. The Creative Commons Public Domain Dedication waiver (http://creativecommons.org/publicdomain/zero/1.0/) applies to the data made available in this article, unless otherwise stated. 


\section{Background}

Autism Spectrum Disorders (ASD), also previously referred to as Pervasive Developmental Disorders (PDD), are a group of neurodevelopmental disorder with onset in early childhood characterized by a triad of core symptoms: impaired social interaction, compromised language and communication and repetitive and narrow pattern of behaviors and interests. Many neuropsychiatric conditions and symptoms are often present in addition to core symptoms in ASD; the most common being mental retardation, hyperactivity, epilepsy, aggressive behaviours. Autism or Autistic Disorder (AD), is the most common and well-known form of ASD that includes Asperger Syndrome (AS), Pervasive Developmental Disorder Not Otherwise Specified (PDD NOS), Childhood Disintegrative Disorder (CDD) and Rett Syndrome (RS). All above subtypes are falling under the single diagnostic category of autism spectrum disorders, according to the new revision of the Diagnostic and Statistical Manual of Mental Disorders (DSM-5) [1]. AS is a less severe variant of $\mathrm{AD}$, in which there is no cognitive deficit or language impairment. PDD - NOS defines a subtype of AD that characterizes subjects who are on the autism spectrum but do not fully meet the criteria for other $\mathrm{AD}$ or other ASD, and it was considered a separate diagnostic category in the DSM-IV. CDD and RS are more rare forms of ASD and show more distinct features from the rest of ASD [2]. CDD is a rare and not very well-known variant of ASD characterized by normal development followed by marked regression in many skills that begin after the age of two [3, 4]. Rett Syndrome (RS) is caused in $80 \%$ of cases by sporadic mutations of MECP2 located on $\mathrm{Xq} 28$. As opposed to AD, RS is more common in females. It has a lifetime prevalence of about 1 in 20,000 females and is characterized by apparently normal development for the first 6-18 months of life, followed by a period of regression in language and motor skills [5]. Both CDD and RS represent nosologically distinct disorders from $\mathrm{AD}, \mathrm{AS}$ and PDD - NOS. In this manuscript, we use the term ASD to refer to AD, AS and PDD-NOS since the three diagnoses can be considered as a continuum rather than clear distinct disorders, consistently with the newly revised DSM-5. Within each of the three diagnostic categories, clinical heterogeneity is substantial. The fact that $\mathrm{AD}$ children share the same diagnostic categories does not imply they share set or severity of core and ASD-associated symptoms. Also, heterogeneity on ASD aetiology is more than likely. Several etiological hypotheses exist, such as altered synaptic dysfunction [6] leading to imbalanced excitatory and inhibitory neurotransmission [7]. However, a unifying aetiological theory is still lacking, and it is more than likely that the clinical heterogeneity is also paralleled by etiological heterogeneity.

Prevalence of ASD is reported to be much higher in most recent studies as compared to studies conducted a decade ago. Recent epidemiological studies indicate a worldwide prevalence for ASD ranging from 0.6 to $0.7 \%$ with an approximate males to females ratio of $4-5$ to 1 $[8,9]$, as compared to the prevalence of less than $0.2 \%$ in earlier investigations [10]. The interpretation of increased prevalence detected in recent studies is not straightforward and has raised controversial debates on the potential causes. The original paper published in Lancet [11] that initiated the controversy on vaccines as a putative cause of autism has been recently retracted by the Journal since fraud has been detected. The vast majority of the scientific community agrees on the unlikelihood of vaccination as a cause. One confounding factor, when reporting prevalence estimates, could be the change over time of diagnostic criteria for ASD, making the comparisons of epidemiological studies more difficult. It is commonly believed that the increase in the overall reported prevalence is mostly due to the development of more comprehensive ascertainment and to the expansion of diagnostic categories, which include milder forms of the disorder [12].

Considerable evidence from twin studies indicates a substantial genetic component in the aetiology of ASD. ASD heritability has been estimated to be between 80 and $90 \%$ according to recent family and twin studies [13]. Monozygotic twin concordance has been found to as high as $>88 \%$, whilst in dizygotic pairs and siblings the concordance rates are $>30 \%$ and $>15 \%$, respectively, higher than what previously reported, suggesting a significant environmental contribution to ASD [14]. Substantial heterogeneity also exists for the genetic component of ASD, where the interplay between commonly inherited variants (single nucleotide polymorphism or SNPs) and highly penetrant rare variations results with a complex genetic architecture [15]. A small proportion of ASD cases has been associated to de novo copy number variations (CNV), including deletions and duplications, as well as to de novo pathogenic single nucleotide variations (SNVs), accounting for a total of $3 \%$ of cases according to recent estimates [16]. Furthermore, ASD related syndromes for which the genetic cause is known account for additional $3.4 \%$ of ASD. These data support a polygenic model, where most ASD liability resides with many common variations along the genome, although the total genetic contribution to ASD (including de novo variations, syndromic mutations, non-additive and additive SNP effects) does not exceed $60 \%$, with the remaining proportion being unaccounted for [16]. Still, the increased number of rare variants exhibiting large individual effect sizes leaves open the single major gene model, consistent with earlier estimates suggesting about 10 to $20 \%$ of ASD cases to be due to known genetic abnormalities [17].

The most common ASD associated syndromes include: i) Fragile $\mathrm{X}$ syndrome (caused by expansion of 
polymorphic CGG repeat upstream of the FMR1 gene) in which over $40 \%$ show Autism core symptoms; ii) Angelman syndrome, determined by a deletion of $15 \mathrm{q} 11-$ 13 where ASD is present in about $50 \%$ of cases; iii) Tuberous Sclerosis, due to mutations in two functionally-related genes, TSC1 and TSC2, in which autism is present in more than 60\% cases; iv) 22q deletion (or Phelan-McDermid) syndrome where about $80 \%$ of cases present with autistic symptoms [16].

Recent sequencing approaches (including exome- or whole-genome sequencing) have contributed to the identification (or validation) of highly penetrant rare genetic variants, including CHD8, GRIN2B, SCN2A, and SYNGAP1 [18] (see also [19] for a recent review). De novo mutations associated to ASD are often located in genes functionally correlated. Among them, neuroligin 3 and 4 (NLGN3 and NLGN4), Neurexin3, SHANK3 and CNTNAP2, which are all involved in synapse formation and specialization, supporting the notion that altered synaptic formation can lead to abnormal neural pathways in ASD) [6]. However, it is important to underline that no single neurobiological hypothesis yet can explain the aetiology of ASD, which is still elusive and likely to be determined by multiple genetic and non-genetic factors.

As far as the contribution from commonly inherited variants, the advent of affordable high-throughput genotyping technologies allowing for genome-wide association studies (GWAS), combined with the increased availability of ASD collections, is beginning to pay-off. Although limited in statistical power to robustly identify genome-wide significant loci, early studies have shown the potential of GWAS in the identification of putative novel risk variants [20]. Two of the first GWAS in ASD reported the identification of common genetic variants residing between two genes mapping on the chromosome 5 and coding for cell molecule adhesion proteins (cadherin 9 and cadherin 10), providing support to a previously hypothesized altered neuronal cell adhesion in ASD [21, 22]. A third study, based on a broad collaborative effort, lead to the identification of an additional common variant on chromosome 5 , adjacent to several genes including SEMA5A, a member of semaphorin protein involved in axonal guidance [23]. Later, the Autism Genome Project (AGP, The AGP Consortium, representing more than 50 centers in North America and Europe) identified genome-wide association signals for SNPs located in the gene MACROD2 [24] and CNTNAP2, a gene previously implicated in ASD [25]. Most of the above findings were not replicated in a subsequent meta-analysis in European samples [26, 27], suggesting the need for larger ASD cohorts to clarify the role of common variation in the disorder. Large-scale coordinated international collaborations such as the Psychiatry Genomic Consortium (PGC) have been established, with the aim of conducting statistically rigorous and comprehensive GWAS meta-analyses for major psychiatric disorders $[28,29]$. The first meta-analysis of the ASD Working Group of the Psychiatric Genomics Consortium, conducted on over 16,000 ASD individuals has been recently published [30]. The study did not show individual variants exceeding the accepted GWAS threshold in the discovery dataset. However, it was able to identify a novel and genome-wide significant association on chromosome 10q24.32 (mapping in CUEDC2) in the combined meta-analysis and confirmed among top-ranking associations some of the findings from previous GWAS.

Notwithstanding the impact of modern genome-wide approaches in the dissection of the complex genetic component of ASD, the vast majority of it is still to be unveiled. The analysis of larger and, in particular, clinically well-characterized ASD samples is critical for the identification of new genetic variants and their validation, and for establishing of genotype-phenotype correlations. With these premises, the 'Fondazione Smith Kline' has supported the creation of an area for autism research that has launched the Italian Autism Network (ITAN) initiative. This initiative aims to create a repository of biological material (DNA, RNA and plasma) and a database of clinical information on autism individuals and their first-degree relatives enabling research on the aetiology and phenomenology of ASD. The recruitment of the first families began back in 2008. In this article, we describe the protocol used by all recruiting sites for clinical evaluation of the ASD subjects and relatives. We also provide the main clinical features of the 249 recruited families.

\section{Methods \\ Study design}

The 'Fondazione Smith Kline' Italy, with its "Autism Research Area", subsequently spun out as a separate Foundation ("ITAN - Italian Autism Network"), has sponsored a multi-centric project to recruit and assess autism individuals and their first-degree relatives to create a repository of genomic DNA, plasma, peripheral RNA and lymphoblastoid cell lines (LCLs). Extensive clinical data and medical history of autistic subjects and their first-degree relatives have also been stored in the database. The goal of the project is to build a biorepository that interacts with a database of extensive clinical information collected electronically at each recruiting sites, enabling genetic, genomic and proteomic research on ASD. Although the target research areas are multidisciplinary (from genetic and biomarker research, to epidemiological studies), the design of the project was driven mainly by its genetic goals. The project aims at collecting small families formed by a proband (ASD child), a sibling when available, and their parents. The collection of families would allow 
performing family-based genetic association studies as well as the screening of genetic variants in cases and affected and unaffected first-degree relatives.

\section{Recruiting sites and ethical issues}

The biorepository is hosted by the Department of Neurosciences, Biomedicine and Movement Sciences at the University of Verona, Italy. Recruitment has begun in 2008 and has been conducted thus far at thirteen different Italian sites (Verona, Acireale (Catania), Bari, Bologna, Brescia, Cagliari, Lecco, Napoli, Padova, Pisa, Rimini, Roma, and Troina (Enna).

Approximately half (48\%) of the patients recruited were referred by the primary care physician, $31 \%$ by a tertiary care center, and the rest by schools, or outpatient clinics.

A study protocol for the clinical assessment and sample management was written and agreed by the main centers and founding members of the network. Candidate recruiting sites were selected among clinical centers with a demonstrated ability to assess autism families as described in the project protocol. The study and its protocol were approved by the Azienda Ospedaliera Universitaria Integrata of Verona Ethic Review Board. Furthermore, each recruiting site obtained independent approval to conduct the project by their respective local ethical review committees. Eighteen sites joined the Italian Autism Network, with thirteen actively recruiting. All adult subjects participating in this project gave their written consent (or the consent for their chidren) to donate biological samples and clinical and demographic information to participate in this study; assent to participate to this study from the children was obtained whenever possible.

\section{Subjects}

Cases, parents and, whenever available, sibling and/or non-affected individuals identified as controls, were recruited. Based on the selection criteria and the family composition, different type of families can be summarized through the pedigree analysis. We used the $\mathrm{R}$ package kinship2 with the pedigree shrinking function to gain a metric of pedigree structure in terms of bitSize (a measure is defined as 2 * \# NonFounders - \# Founders, see [31]).

\section{Inclusion and exclusion criteria}

A proband child or affected sibling was eligible for recruitment when all the following inclusion criteria apply: 1 ) at least 4 years of age at the time of the interview, 2) having at least one parent or legal guardian giving voluntary written consent for their children to take part to the research project, 3) meeting DSM-IV criteria for Autistic Disorder or Asperger's Disorder, or Pervasive Developmental Disorder Not Otherwise Specified (PDD-NOS),
4) reaching score cut-off in ADI-R. Siblings of the proband were recruited when available, and defined as unaffected, if they met the following criteria: 1) at least 4 years of age at the time of recruitment, 2) devoid of any comorbid psychiatric illness as determined by: i) the Child Behavioural Checklist (CBCL) or by the Kiddie Schedule for Affective Disorders and Schizophrenia for children (Kiddie-SADS), ii) absence of social skills impairment as determined by the Social Responsiveness Scale (SRS), iii) the Broader Phenotype Autism Symptom Scale (BPASS) (administered to evaluate the presence of autism spectrum symptoms). Individuals were not eligible for recruitment if any of the following criteria apply: 1) history of serious head injury, encephalitis or tumors, 2) age above 18; 3) Diagnoses of Childhood Disintegrative Disorder or Rett Syndrome, diagnosis of known ASD-related genetic syndromes or presence of severe mental retardation $(\mathrm{IQ}<20)$ that would compromise the validity of the diagnosis of autism.

\section{Clinical assessment}

Diagnosis was established by experienced child psychiatrists after completing full neurological, psychiatric and physical examination, and after reviewing and completing the Autism Diagnostic Interview - Revised (ADI-R) and the Autism Diagnostic Observation Schedule (ADOS) that were administered by trained and certified clinicians. The cognitive level of children was assessed whenever possible through either one of the following scales: Wechsler Intelligence Scale for Children, Wechsler Preschool and Primary Scale of Intelligence, Leiter International Performance Scale or Griffiths Mental Developmental Scale. Additional structured evaluation included the Children's Global Assessment Scale (CGAS) and, for subjects with suspect of Asperger's Disorder, the Krug Asperger's Disorder Index (KADI). The KADI is an individually administered, norm-referenced screening instrument that provides useful information for determining the diagnosis of Asperger's Disorder. It is standardized for use with individuals 6 through 21 years of age. Instrumental and laboratory examination of the fragile X (FRAX) expansions and major cytogenetic abnormalities were conducted for all the autism individuals and their siblings. MRI scan and awake and nap EEG were evaluated in all patients, if they were not available from previous assessments conducted at a close time.

Parents of the probands and siblings of age 18 and above were assessed as follows: i) Cognitive level: four items of the WAIS (arithmetic, vocabulary, block design, picture arrangement); ii) Language: Token test; iii) Autism spectrum symptoms: BPASS Broader Phenotype Autism Symptom Scale (BPASS); iv) Psychopathology: SCID screening interview. 
Non-affected siblings of less and 18 years of age recruited were assessed with medical, psychiatric and neurologic examination. For each non-affected sibling, cognitive levels are established via the four items of the Wechsler Intelligence Scale for Children (WISC) that has shown to produce a reliable measure of IQ [32]. The four items include the arithmetic, vocabulary, block design, and picture arrangement. Language competence was assessed with the Peabody Picture Vocabulary test (PPVT-R), for ages between 4 and 12, and the Token Test for age between 12 and 18. Social Responsiveness Scale (SRS) was also administered to evaluate socialization. Psychopathology in non-affected siblings was assessed via the Child Behavioural Checklist (CBCL). The following definitions were used to define the presence of psychopathology: T-score: normal <65; at risk: 66-74; pathological: $>75$ (2.5 SD from normal average) if scores less above 75 (presence of psychopathology) the Kiddie-SADS interview is administered to diagnose the psychiatric interview.

\section{Sample collection, handling and storage}

The Italian Autism Network collected DNA, plasma, RNA and EBV transformed lymphocytes in the biorepository from each of the individuals assessed. From all individuals eligible to participate in this project (as either cases or unaffected relatives) for whom a signed informed consent was obtained, the following peripheral blood samples were taken: 1) $2 \times 6-\mathrm{mL}$ in ACD blood tubes for DNA extraction; 2) $1 \times 6 \mathrm{~mL}$ ACD tube for extracting lymphocytes to be transformed with Epstein Barr virus (EBV) in LCLs; 3) $1 \times 6-\mathrm{mL}$ in EDTA tube to obtain plasma aliquots for proteomic analysis, and 4) $2 \times 2.5-\mathrm{mL}$ in PAX gene tubes for obtaining RNA. Plasma aliquots $(0.2 \mathrm{~mL})$ were prepared by centrifugation of EDTA tubes and stored at $-80{ }^{\circ} \mathrm{C}$. Blood-derived samples for RNA extraction were prepared by gently mixing PAX gene tubes, storing them for $2 \mathrm{~h}$ at room temperature, cooling them at $-20^{\circ}$ and later at $-80{ }^{\circ} \mathrm{C}$ to be ready for shipment. Samples for DNA extraction and cell lines transformation were shipped at room temperature to the biorepository on the same day of collection. Frozen samples (plasma, blood samples for RNA) were shipped via express courier in dry ice to the biorepository.

Upon delivery at the biorepository, Plasma aliquots and PAX gene tubes were stored at $-80{ }^{\circ} \mathrm{C}$. DNA extraction was performed using the Puregene Blood Kit (Gentra Systems, Minneapolis, MN, US), a modified salting-out precipitation method, following the manufacturer's instructions. Each DNA sample was then quality controlled and quantified using NanoDrop ND 1000 spectrophotometer (Thermo Scientific, Wilmington, DE, US). RNA samples from lymphocytes were isolated with QIAcube system, quantified with NanoDrop and analyzed for quality control by use of the RNA 6000 NanoLabChip (Agilent Technologies, Santa Clara, CA, US). The biorepository also transformed the lymphocytes with EBV into lines that were and then stored in Liquid

Table 1 Distribution of subjects among recruiting centers and summary of demographic information

\begin{tabular}{|c|c|c|c|c|c|c|c|c|c|c|c|c|}
\hline \multirow[t]{3}{*}{ Site } & \multirow[t]{3}{*}{ Families } & \multirow[t]{3}{*}{ Parents } & \multirow{3}{*}{$\begin{array}{l}\text { Unaffected } \\
\text { siblings }\end{array}$} & \multicolumn{9}{|c|}{ Autism Spectrum Disorders } \\
\hline & & & & \multirow{2}{*}{$\begin{array}{l}\text { ASD } \\
\text { cases }\end{array}$} & \multirow{2}{*}{$\begin{array}{l}\text { Gender } \\
(\% \\
\text { males) }\end{array}$} & \multirow{2}{*}{$\begin{array}{l}\text { Average } \\
\text { Age (SD) }\end{array}$} & \multicolumn{3}{|c|}{ Ethnicity } & \multicolumn{3}{|c|}{ Diagnosis } \\
\hline & & & & & & & AFR & OTH & CEU & $\overline{A D}$ & ASPG & PDD-NOS \\
\hline 3 & $66^{\mathrm{b}}$ & 122 & 57 & 68 & 85.29 & $9.44(3.84)$ & 8 & 0 & 60 & 50 & 9 & 9 \\
\hline 4 & $4^{\mathrm{b}}$ & 8 & 0 & 5 & 100.00 & $10.60(1.67)$ & 0 & 0 & 5 & 3 & 0 & 2 \\
\hline 5 & $27^{a}$ & 51 & 1 & 26 & 92.31 & $5.38(2.17)$ & 0 & 2 & 24 & 18 & 0 & 8 \\
\hline 6 & $21^{\mathrm{b}}$ & 41 & 7 & 22 & 72.73 & $9.45(4.68)$ & 11 & 0 & 11 & 18 & 1 & 3 \\
\hline 7 & 17 & 33 & 10 & 17 & 82.35 & $10.71(4.55)$ & 8 & 3 & 6 & 13 & 3 & 1 \\
\hline 8 & $32^{\mathrm{a}}$ & 51 & 0 & 31 & 83.87 & $6.71(2.77)$ & 16 & 0 & 15 & 5 & 2 & 24 \\
\hline 9 & 17 & 31 & 11 & 17 & 88.24 & $11.24(4.12)$ & 0 & 0 & 17 & 11 & 1 & 5 \\
\hline 10 & $8^{\mathrm{b}}$ & 16 & 1 & 9 & 88.89 & 7.89 (3.95) & 2 & 0 & 7 & 6 & 0 & 3 \\
\hline 11 & 13 & 23 & 2 & 13 & 76.92 & $11.77(4.55)$ & 4 & 0 & 9 & 4 & 6 & 3 \\
\hline 12 & $11^{\mathrm{b}}$ & 22 & 3 & 12 & 75.00 & $5.25(1.71)$ & 0 & 0 & 12 & 4 & 0 & 8 \\
\hline 13 & $9^{a}$ & 15 & 2 & 8 & 87.50 & $10.88(2.85)$ & 0 & 1 & 7 & 5 & 1 & 2 \\
\hline 16 & 4 & 8 & 3 & 4 & 100.00 & $10.50(5.51)$ & 0 & 0 & 4 & 4 & 0 & 0 \\
\hline 17 & 20 & 40 & 2 & 20 & 95.00 & $10.05(4.67)$ & 2 & 0 & 18 & 12 & 2 & 6 \\
\hline Tot & 249 & 461 & 99 & 252 & 86.78 & $9.22(3.62)$ & 51 & 6 & 195 & 153 & 25 & 74 \\
\hline
\end{tabular}

Distribution of subjects among recruiting centers and summary of demographic information

andicates centers where family recruitment is not completed (eg affected subjects less than $\mathrm{N}$ of families)

${ }^{b}$ Indicates centers which have recruited families with $>1$ affected subjects 


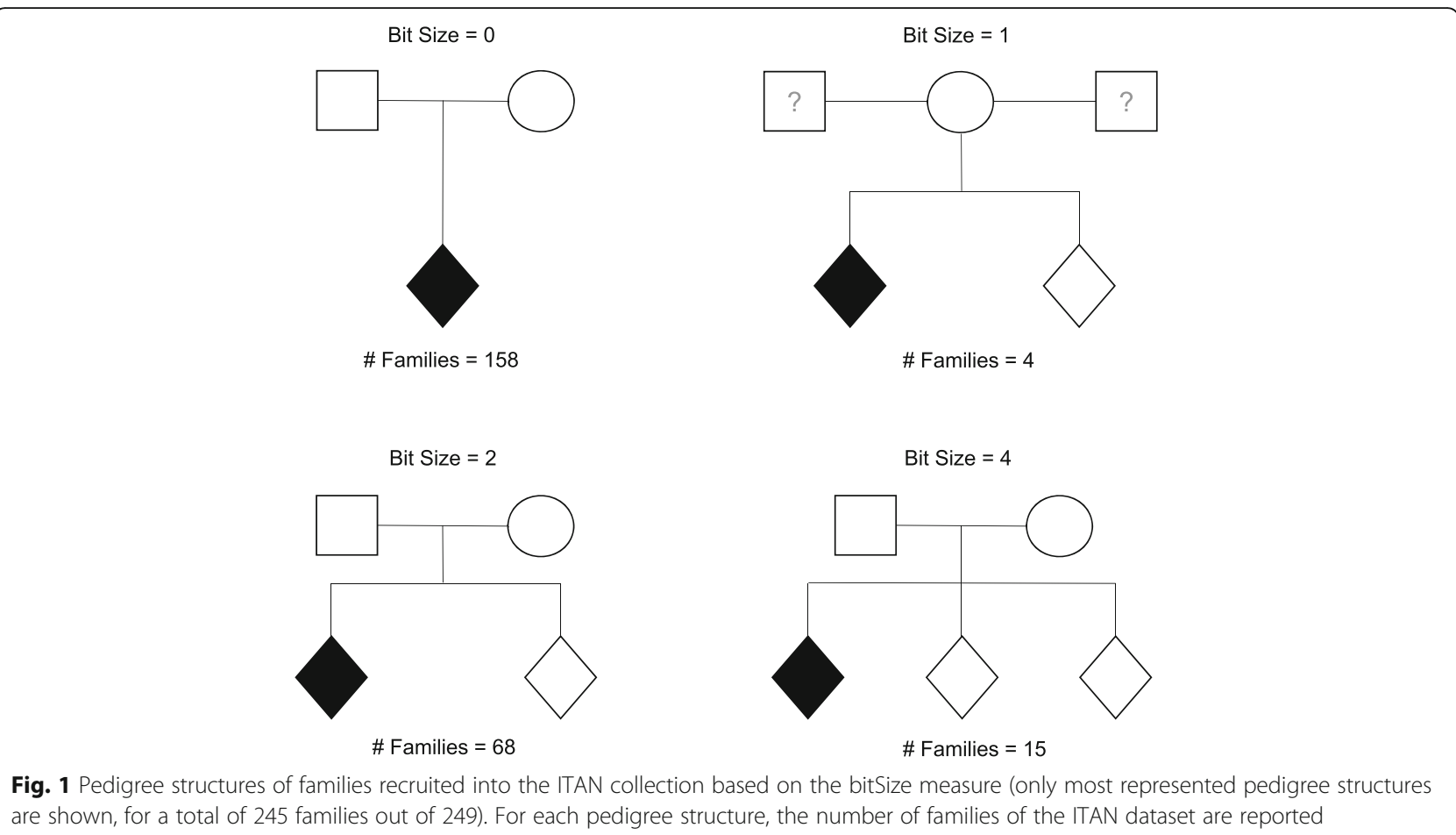

Nitrogen. All relevant data regarding the samples are entered into a computerized system that allows matching the information of the samples with the clinical data stored in the centralized clinical database.

\section{Data security procedure for the clinical database}

All blood, plasma, cell lines, DNA and RNA samples are coded and stored under secure conditions with restricted access according to the Italian privacy policies required for keeping secure sensitive data. The clinical information collected at each site is entered into an encrypted system and stored in a database. Codes corresponding to the research subjects are known only by the clinician responsible for the research project, who obtained the informed consent from the parents of affected subjects and any sibling at each recruiting site (Site Principal Investigator), and by the clinical personnel directly involved in the assessment of the research subject, as required by standard clinical practice.

\section{Discussion}

The Italian Autism Network (ITAN) was initiated under the sponsorship of the Fondazione Smith Kline with the aim of creating an open resource for clinical, genetics and biomarker research in autism. Here we describe the protocol used to build up this novel family collection and the associated database and biorepository. Each family is composed by at least one ASD affected child and, when possible, a discordant sibling.

\section{Recruitment progress to date}

To date, the ITAN consortium has recruited 812 subjects, i.e. 252 probands and 560 first degree relatives, for a total of 249 families. In Table 1 we provide information on the distribution of samples among recruiting centers and the demographic of the collection. In Fig. 1 we

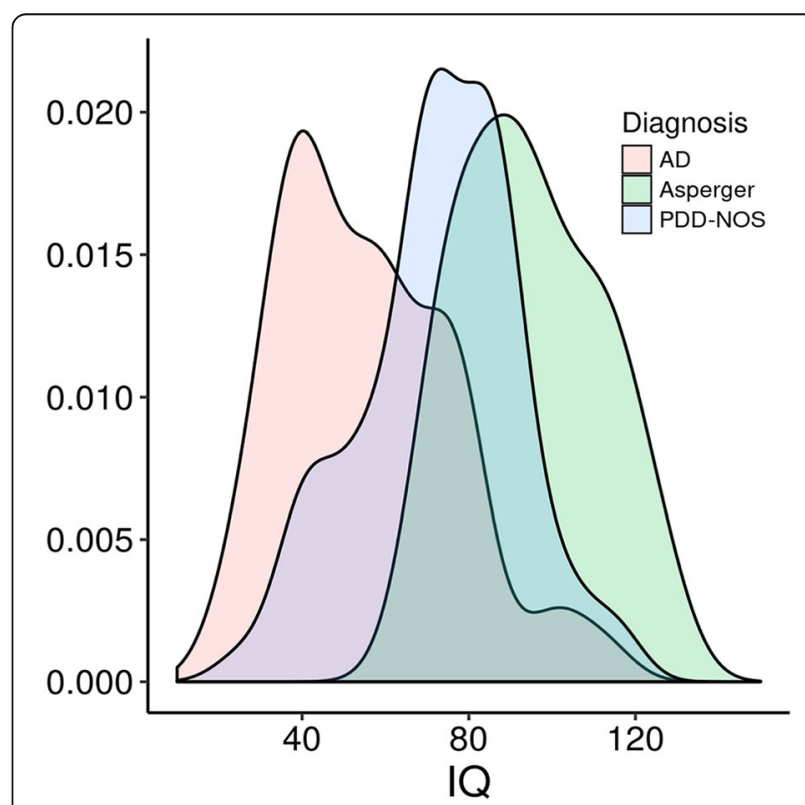

Fig. 2 distribution of the cognitive level of ASD subjects of the ITAN collection, as measured by $1 \mathrm{Q}$ assessments, for each of the three diagnostic categories 
Table 2 Intellectual quotients (IQ) of affected subjects across diagnostic categories

\begin{tabular}{llllll}
\hline Diagnosis & $\mathrm{IQ}<35$ & $\mathrm{IQ}=35-50$ & $\mathrm{IQ}=50-75$ & $\mathrm{IQ}>75$ & not available \\
\hline Autistic Disorders & 17 & 53 & 49 & 29 & 5 \\
Asperger Syndrome & 0 & 0 & 4 & 20 & 1 \\
Pervasive & 1 & 9 & 29 & 33 & 2 \\
Developmental & & & &
\end{tabular}

Disorders

illustrate the distribution of pedigree structures among families recruited in the study.

\section{Clinical phenotypes}

The clinical assessment was derived by full neuropsychiatric evaluation and structured interviews (i.e.: ADI-R, and ADOS) by appropriately trained and certified staff. The average age at interview of the affected and non-affected subjects recruited to date is 8.89 and 11.53 , respectively, while the ratio of males in the affected samples is $85.31 \%$, reflecting the higher incidence of autism in males reported for the disease in the general population. For the non-affected siblings, the proportion of males is $55.55 \%$. Figure 2 reports the distribution of the cognitive level for each diagnostic category (DSM IV), as measured by IQ assessment. As expected, high functioning Asperger subjects have higher values of IQ compared to PDD-NOS and AD subject group (the latter showing the lowest IQ values). Only $7.1 \%$ of the affected subjects $(n=18)$ have serious mental retardation with a low IQ $(<35)$ as shown in Table 2. It should be however noted, that IQ below 20 was an exclusion criterion for the probands.

\section{Preliminary genetic investigations}

So far, DNA samples from subjects of the ITAN collection have been utilized for testing candidate risk variants for autism derived from large GWAS investigations [26, 27, 33]. Furthermore, the regulation of candidate genes has been studied by analyzing RNA expression in LCLs from selected probands and non-affected siblings [34]. A large proportion of the discordant sibling pairs (for a total of 152 samples) was genotyped on the PsychArray (Illumina Inc., San Diego, California, USA) and blood RNA was sequenced on the Illumina HiSeq 2000 RNA Seq Platform. The availability of both genotype and RNA expression data on a genome scale is being exploited for a novel integrative approach aimed at the identification of genetical-genomic biomarker signatures for autism $[35,36]$.

In conclusion, the ITAN collection clearly represents a valuable resource for the identification and characterization of common and rare genetic risk variants for autism, thanks to the accuracy of the clinical assessment and the family design. The availability of different types of biological samples makes it a unique collection for the conduct of biomarkers investigations integrating multiple "omic" platforms.

\section{Abbreviations}

AD: Autistic Disorder; ADI-R: Autism Diagnostic Interview-Revised; ADOS: Autism Diagnostic Observation Schedule; ASD: Autism Spectrum Disorder; BPASS: Broader Phenotype Autism Symptom Scale; CBCL: Child Behavioural Checklist; CGAS: Children's Global Assessment Scale; DSMIV: Diagnostic and Statistical Manual of Mental Disorders, 4TH Edition, Text Revision; EEG: Electroencephalography; FRAX: Fragile X; GWAS: Genome-Wide Association Study; IQ: Intelligent Quotient; ITAN: Italian Autism Network; KADI: Krug Asperger's Disorder Index; Kiddie-SADS: Kiddie Schedule for Affective Disorders and Schizophrenia for children; LCL: Lymphoblastoid Cell Lines; PDD-NOS: Pervasive Developmental Disorder Not Otherwise Specified; PPVT-R: Peabody Picture Vocabulary Test - Revised; SRS: Social Responsiveness Scale; WAIS: Wechsler Adult Intelligence Scale; WISC: Wechsler Intelligence Scale for Children

\section{Acknowledgments}

We would like to thank first of all family members who provided their time and samples that made this collection possible.

We would also like to thank the following individual who contributed to the creation of ITAN:

Leonardo Zoccante, Flavio Boscaini. Servizio di Neuropsichiatria Infantile Azienda Ospedaliera Istituti Ospitalieri di Verona Policlinico G.B. Rossi, Verona. Maurizio Elia, Giuseppa Di Vita, IRCCS "Associazione Oasi Maria SS." (Istituto di Diritto Privato), Troina (Enna).

Massimo Molteni, Elisa Mani, Elisa Ceppi, IRCCS "Eugenio Medea", Monza. Antonio Pascotto, Carmela Bravaccio Neuropsichiatria Infantile - Seconda Università degli Studi di Napoli.

Alessandra Tiberti, Filippo Gitti, Giovanni Allibrio, Azienda Ospedaliera Spedali Civili di Brescia. Brescia.

Lucia Margari, Anna Linda Lamanna, Andrea DeGiacomo, Unità Operativa di Neuropsichiatria Infantile Dipartimento di Scienze Neurologiche e Psichiatriche Università degli Studi Bari, Bari.

Alessandro Zuddas, Laura Anchisi, Università degli Studi di Cagliari - Centro per lo Studio delle Terapie Farmacologiche in Neuropsichiatria dell'infanzia e dell'adolescenza, Cagliari.

Paolo Curatolo, Barbara Manzi, Arianna Benvenuto, Clinica S.Alessandro Cattedra di Neuropsichiatria Infantile - Università "Tor Vergata" - Policlinico Tor Vergata, Roma. Gabriele Masi, Raffaella Tancredi, IRCCS Stella Maris, Pisa. Franco Nardocci, Serenella Grittani, Tiziana Piroddi, Milena Andruccioli,

Ospedale Infermi - Divisione Neuropsichiatria Infantile - Centro per l'autismo, Rimini.

For the biorepository we thank Pier Franco Pignatti, Elisabetta Trabetti, Paola Prandini.

For the executive group we thank Diego Cosentino, Sonia Colcera (GSK), Maurizio Bassi (Fondazione Smith Kline), Luigi Napolitano (Fondazione Smith Kline), Francesca Arzone (GSK), and Ugo Bianchi and Maurizio Martignano from Ad-hoc Sistemi. We would like to acknowledge the multidisciplinary Scientific Committee of the Fondazione SmithKline "Area Ricerca Autismo" that guided the project since its conception and regularly monitored its progression for ethical and scientific rigor.

ITAN (the Italian Autism Network) is composed by: Giovanni, Alibrio; Laura, Anchisi; Milena, Andruccioli; Arianna, Benvenuto; Pier Antonio, Battistella; Flavio, Boscaini; Carmela, Bravaccio; Elisa, Ceppi; Diego, Cosentino; Paolo, Curatolo; Lucio, Da Ros; Bernardo, Dalla Bernardina; Andrea, De Giacomo; Giuseppa, Di Vita; Enrico, Domenici; Massimo, Elia; Filippo, Gitti; Serenella, Grittani; Anna Linda, Lamanna; Elisa, Mani; Barbara, Manzi; Lucia, Margari; Gabriele, Masi; Massimo, Molteni; Pierandrea, Muglia; Franco, Nardocci; 
Antonio, Pascotto; Antonia, Parmeggiani; Pier Franco, Pignatti; Tiziana, Piroddi; Paola, Prandini; Emiliangelo, Ratti; Paolo, Rizzini; Sebastiano, Russo; Renato, Scifo; Raffaella, Tancredi; Alessandra, Tiberti; Elisabetta, Trabetti; Leonardo, Zoccante; Alessandro, Zuddas.

\section{Funding}

The study was funded by Fondazione Smith Kline and the Italian Ministry of Education, University and Research. The funding bodies had no role in the study design, data collection and analysis, decision to publish, or preparation of the manuscript.

\section{Availability of data and materials}

The ITAN is an open resource. In 2012 the Fondazione Smith Kline transferred the ownership to a newly created not-for-profit organization 'Italian Autism Network' Foundation that govern the use of the resource with appropriate governance (see ITAN Web site: https://www.fondazioneitan.org for details). To gain data access, interested researchers should submit an official request to the Foundation. Data will be released only for specific research purposes, upon a positive assessment of a project proposal by the ITAN Scientific Committee.

\section{Authors' contributions}

PM, LDR, PR, BDB, ER designed the study, wrote the protocol and coordinated the implementation of the network, its infrastructure and logistics. ET has responsibility for the biorepository and was actively involved in writing the sample collection and shipping guidelines. MF, TKT, LDR, PM and $E D$ wrote the manuscript. BDB, ET, FN, AZ, ER, PM, ED are members of the Scientific Committee, reviewed and approved the manuscript.

\section{Ethics approval and consent to participate}

The study protocol was in first instance approved by the Verona Hospital Ethical Review board (study protocol AUT-SFK001, CE1419), and subsequently by following Ethical Review Committees of each recruiting site:

- Comitato Etico per la Sperimentazione, Azienda Ospedaliera di Verona, Verona, Italy

- Comitato Etico dell'Azienda Ospedaliera Universitaria, Seconda Università degli studi di Napoli, Napoli, Italy

- Comitato Etico di Area Vasta Romagna ed. IRST, Meldola (FC), Italy

- Comitato Etico Indipendente locale, Azienda Ospedaliera "Ospedale Policlinico Consorziale" di Bari, Bari, Italy

- Comitato Etico Azienda Ospedaliera Spedali Civili di Brescia, Brescia, Italy

- Comitato Etico Azienda Ospedaliero Universitaria di Cagliari, Cagliari, Italy

- Comitato Etico IRCCS Associazione Oasi Maria SS. ONLUS, Troina (EN), Italy

- Comitato Etico Indipendente PTV Azienda Ospedaliera Universitaria, Policlinico di Tor Vergata, Roma (Italy)

- Comitato Etico Associazione La Nostra Famiglia IRCCS E. Medea, Bosisio Parini (LC), Italy

- Comitato Etico di Fondazione Stella Maris, Calambrone (PI), Italy

- Comitato Etico Indipendente Servizio Sanitario Regionale Emilia Romagna, Azienda Unitaria Sanitaria Locale di Bologna, Italy

- Comitato Etico per la Sperimentazione Azienda Ospedaliera di Padova, Padova, Italy

- Comitato Etico Azienda Sanitaria Provinciale Catania, Catania, Italy

All adult subjects participating in this project gave their written consent (or the consent for their chidren) to donate biological samples and clinical and demographic information to participate in this study; assent to participate to this study from the children was obtained whenever obtainable. A multidisciplinary Scientific and Ethical Committee of the Fondazione SmithKline, "Area Ricerca Autismo", i) created the protocol; ii) ensured that the use of the samples and clinical database was consistent with what consented by the research participants; iii) ensured that research was conducted with sound methodology and by accredited institutions.

\section{Consent for publication}

Not applicable.

\section{Competing interests}

PM is an employee of UCB Pharma (Belgium); ER is an employee of Takeda (USA); TKT is an employee of F. Hoffman-La Roche Ltd. (Switzerland); PR is an employee of ViiV Healthcare. PM, ED, LDR and ER were employees of GSK (Italy) during the design and launch of the clinical study. ED has received research support from Roche in the period 2016-2018. AZ has served on the advisory boards of Shire, EcuPharma and Angelini and on data safety monitoring boards of Otsuka and Lundbeck; he has received research support from Shire, Vifor, Roche, Lundbeck, Jannssen.

\section{Publisher's Note}

Springer Nature remains neutral with regard to jurisdictional claims in published maps and institutional affiliations.

\section{Author details}

${ }^{1}$ UCB Pharma, Bruxelles, Belgium. ${ }^{2}$ Centre for Integrative Biology, University of Trento, Trento, Italy. ${ }^{3}$ Fondazione Smith Kline, Verona, Italy. ${ }^{4}$ Roche Pharmaceutical Research and Early Development (pRED), Roche Innovation Center, Grenzacherstrasse 124, Basel, Switzerland. ${ }^{5}$ Neuropsichiatria infantile, Modena, Italy. ${ }^{6}$ Department of Neurosciences, Biomedicine and Movement Sciences, Section of Biology and Genetics, University of Verona, Verona, Italy. ${ }^{7}$ Central Nervous System (CNS) Therapeutic Area Unit, Takeda, Boston, USA. ${ }^{8} \mathrm{Child}$ and Adolescent Psychiatry Unit, Department of Biomedical Sciences, University of Cagliari, Cagliari, Italy. ${ }^{9}$ Servizio di Neuropsichiatria Infantile Azienda Ospedaliera Istituti Ospitalieri di Verona Policlinico G.B. Rossi, Verona, Italy. ${ }^{10}$ The Microsoft Research - University of Trento Centre for Computational and Systems Biology, Rovereto, TN, Italy.

Received: 26 April 2018 Accepted: 23 October 2018

Published online: 21 November 2018

\section{References}

1. American Psychiatric Association. Diagnostic and statistical manual of mental disorders. 5th ed. Washington, DC: American Psychiatric Association; 2013

2. Hendry CN. Childhood disintegrative disorder: should it be considered a distinct diagnosis? Clin Psychol Rev. 2000;20:77-90

3. Volkmar FR. Childhood disintegrative disorder: issues for DSM-IV. J Autism Dev Disord. 1992;22:625-42.

4. Weiss LA, Arking DE, Gene Discovery Project of Johns Hopkins \& the Autism Consortium, Daly MJ, Chakravarti A. A genome-wide linkage and association scan reveals novel loci for autism. Nature. 2009;461:802-8.

5. Chahrour M, Zoghbi HY. The story of Rett syndrome: from clinic to neurobiology. Neuron. 2007;56:422-37.

6. Bourgeron T. From the genetic architecture to synaptic plasticity in autism spectrum disorder. Nat Rev Neurosci. 2015;16:551-63.

7. Nelson SB, Valakh V. Excitatory/inhibitory balance and circuit homeostasis in autism Spectrum disorders. Neuron. 2015;87:684-98.

8. Elsabbagh M, Divan G, Koh YJ, Kim YS, Kauchali S, Marcín C, et al. Global prevalence of autism and other pervasive developmental disorders. Autism Res. 2012;5:160-79.

9. Lai MC, Lombardo MV, Baron-Cohen S. Autism. Lancet. 2014;383:896-910.

10. Fombonne E. The epidemiology of autism: a review. Psychol Med. 1999; 29:769-86.

11. Wakefield AJ, Murch SH, Anthony A, Linnell J, Casson DM, Malik MA, et al. lleal-lymphoid-nodular hyperplasia, non-specific colitis, and pervasive developmental disorder in children. Lancet. 1998;351:637-41 [retracted].

12. Rutter M. Incidence of autism spectrum disorders: changes over time and their meaning. Acta Paediatr. 2007;94:2-15.

13. Sandin S, Lichtenstein P, Kuja-Halkola R, Hultman C, Larsson H, Reichenberg A. The heritability of autism Spectrum disorder. JAMA. 2017;318:1182-4.

14. Frazier TW, Thompson L, Youngstrom EA, Law P, Hardan AY, Eng C, Morris N. A twin study of heritable and shared environmental contributions to autism. J Autism Dev Disord. 2014;44:2013-25.

15. Gaugler T, Klei L, Sanders SJ, Bodea CA, Goldberg AP, Lee AB, et al. Most genetic risk for autism resides with common variation. Nat Genet. 2014:46:881-5.

16. de la Torre-Ubieta L, Won H, Stein JL, Geschwind DH. Advancing the understanding of autism disease mechanisms through genetics. Nat Med. 2016;22:345-61. 
17. Abrahams BS, Geschwind DH. Advances in autism genetics: on the threshold of a new neurobiology. Nat Rev Genet. 2008;9:341-55.

18. De Rubeis S, He X, Goldberg AP, Poultney CS, Samocha K, Cicek AE, et al. Synaptic, transcriptional and chromatin genes disrupted in autism. Nature. 2014;515:209-15.

19. Vorstman JAS, Parr JR, Moreno-De-Luca D, Anney RJL, Nurnberger JI Jr, Hallmayer JF. Autism genetics: opportunities and challenges for clinical translation. Nat Rev Genet. 2017;18:362-76.

20. Devlin B, Melhem N, Roederb K. Do common variants play a role in risk for autism? Evidence and theoretical musings. Brain Res. 2011;1380:78-84.

21. Wang K, Zhang H, Ma D, Bucan M, Glessner JT, Abrahams BS, et al. Common genetic variants on $5 p 14.1$ associate with autism spectrum disorders. Nature. 2009;459:528-33.

22. Ma D, Salyakina D, Jaworski JM, Konidari I, Whitehead PL, Andersen AN, et al. A genome-wide association study of autism reveals a common novel risk locus at 5p14.1. Ann Hum Genet. 2009;73:263-73.

23. Weiss LA, Arking DE, Daly MJ, Chakravarti A. A genome-wide linkage and association scan reveals novel loci for autism. Nature. 2009;461:802-8.

24. Anney R, Klei L, Pinto D, Regan R, Conroy J, Magalhaes TR, et al. A genomewide scan for common alleles affecting risk for autism. Hum Mol Genet. 2010;19:4072-82.

25. Anney R, Klei L, Pinto D, Almeida J, Bacchelli E, Baird G, et al. Individual common variants exert weak effects on the risk for autism spectrum disorders. Hum Mol Genet. 2012;21:4781-92.

26. Curran S, Bolton P, Rozsnyai K, Chiocchetti A, Klauck SM, Duketis E, et al. No association between a common single nucleotide polymorphism, rs4141463, in the MACROD2 gene and autism spectrum disorder. Am J Med Genet B Neuropsychiatr Genet. 2011;156B:633-9.

27. Torrico B, Chiocchetti AG, Bacchelli E, Trabetti E, Hervás A, Franke B, et al. Lack of replication of previous autism spectrum disorder GWAS hits in European populations. Autism Res. 2017;10:202-11.

28. Sullivan PF. The psychiatric GWAS consortium: big science comes to psychiatry. Neuron. 2010;68:182-6.

29. Sullivan PF, Agrawal A, Bulik CM, Andreassen OA, Børglum AD, Breen G, et al. Psychiatric genomics: an update and an agenda. Am J Psychiatry. 2018; 175:15-27.

30. Consortium Autism Spectrum Disorders Working Group of the Psychiatric Genomics Consortium. Meta-analysis of GWAS of over 16,000 individuals with autism spectrum disorder highlights a novel locus at 10q24.32 and a significant overlap with schizophrenia. Mol Autism. 2017;8:21.

31. Sinnwell JP, Therneau TM, Schaid DJ. The kinship2 R package for pedigree data. Hum Hered. 2014;78:91-3.

32. Wechsler D. Wechsler Intelligence Scale for Children. 4th ed. San Antonio, TX: The Psychological Corporation; 2003.

33. Prandini P, Pasquali A, Malerba G, Marostica A, Zusi C, Xumerle L, et al. The association of rs4307059 and rs35678 markers with autism spectrum disorders is replicated in Italian families. Psychiatr Genet. 2012;22:177-81.

34. Prandini P, Zusi C, Malerba G, Itan, Pignatti PF, Trabetti E. Analysis of RBFOX1 gene expression in lymphoblastoid cell lines of Italian discordant autism spectrum disorders sib-pairs. Mol Cell Probes. 2014:28:242-5.

35. Kam-Thong T, Essioux L, Muglia P, Spooren W, Mueller-Myhsok B, Domenici E, Italian Autism Network (ITAN). Towards novel biomarkers for autism using an integrative genomic approach on discordant sibling pair design. Copenaghen: World Congress Psychiatry Genetics; 2014.

36. Filosi M, Kam-Thong T, Mueller-Myhsok B, Muglia P, Italian Autism Network (ITAN), Domenici E. Blood-based Autism Spectrum Disorder signatures from the Italian Autism Network collection. Orlando: World Congress Psychiatry Genetics; 2017.

\section{Ready to submit your research? Choose BMC and benefit from:}

- fast, convenient online submission

- thorough peer review by experienced researchers in your field

- rapid publication on acceptance

- support for research data, including large and complex data types

- gold Open Access which fosters wider collaboration and increased citations

- maximum visibility for your research: over $100 \mathrm{M}$ website views per year

At $\mathrm{BMC}$, research is always in progress.

Learn more biomedcentral.com/submissions 